\title{
TWO HOPS IS ONE TOO MANY IN AN ENERGY-LIMITED WIRELESS SENSOR NETWORK
}

\author{
Erik Björnemo, Mathias Johansson and Anders Ahlén \\ Signals and Systems, Uppsala University, Box 534, SE-751 21 Uppsala, Sweden. \\ E-mail:\{eb, mj, aa\}@angstrom.uu.se
}

\begin{abstract}
A common technique in wireless sensor networks (WSN) is to use multihopping, that is, relaying messages via intermediate nodes. In this work we compare the energy efficiency of single-hop and multihop taking into account circuit energy consumption as well as transmission energy. We consider a simple two-hop case as well as a multihop case for a uniform two-dimensional network of arbitrary size. Contrary to common beliefs, we find that single-hop is superior for all realistic cases covered by our model. Even in comparison to the simple two-hop case single-hop is preferable. Only at very large path losses would multihop be a serious alternative. At present there are however very few WSN radios available that can operate under such conditions. In spite of the relatively simple networks considered we argue that our findings have quite general applicability with strong implications for the choice of routing protocols.
\end{abstract}

Index Terms - Wireless sensor networks, energy efficiency, multihop, routing

\section{INTRODUCTION}

The transfer of data from a source node to the destination node (the central sink) in a wireless many-to-one sensor network is often presupposed to be carried out in a multihop fashion, that is via intermediate nodes in the network. Naturally, this presupposition is common in the research on routing protocols for wireless sensor networks, see for example [1], but it is also prevalent in the wider research area of energy-efficient wireless sensor networks, see for example [2]. There are however a few studies suggesting that single-hop architectures can be advantageous from an energy perspective, not only because of their simple structure. Min and Chandrakasan [3] have for instance published results regarding the impact of important hardware characteristics on the attractiveness of single-hop networks. They argue that it is a myth that multihop saves energy; by including circuit energy consumption they find that single-hop is often more energy-efficient than multihop, at least in their specific examples. Similar results are also given in [4], and in [5] the authors suggest that proper coding can make single-hop networks more attractive.

In this paper we take a closer look at the energy-efficiencies of multihop and single-hop approaches. More specifically, we first establish some very basic limits for the superiority of a multihop strategy by considering a two-hop structure and then go on to larger networks. Moreover, we show how important system parameters and environment assumptions affect the choice of strategy. We try to avoid restrictive assumptions and the use of specific fixed parameters in order not to bias the comparison unjustly, but for the sake of transparency we deliberately use quite simple models that do not obscure our analysis. Our analysis is in many respects conservative regarding the energy costs for the multihop approach.

\section{COMPARING ENERGY CONSUMPTIONS IN MULTIHOP AND SINGLE-HOP NETWORKS}

The energy metrics we choose for our comparison are both the energy consumed by the most heavily loaded node and the whole network's total energy consumption. The latter has the drawback that it conceals the important effects of asymmetric energy consumption in the network, while these are properly revealed by the former [2]. To clarify this point, consider a large two-dimensional multihop network in which the few nodes closest to the central sink must forward all the data generated in the network. Even if the network as a whole seems energy-efficient it may quickly become dysfunctional as the central nodes run out of energy. In this paper we consider data gathering many-to-one networks where all nodes are identical and perform measurements; the nodes are, in some sense, all considered equally important. ${ }^{1}$ It is therefore appropriate to use the metric of maximum node consumption. A second reason for its application is that fewer assumptions are needed because only one single-hop link in the network needs to be considered; the most energy-demanding one. Therefore, this metric imposes no constraints (assumptions) on the node placement (node distribution).

\subsection{Energy Model and Metric}

We model the per-hop energy consumption per transmitted bit as consisting of three terms: the transmitter circuit energy $E_{c t}$, the receiver circuit energy $E_{c r}$ and the transmission energy $E_{t}$. The former is the energy consumed by the node circuitry involved in processing each bit before transmission, for example the digital-toanalog converter, the local oscillator and the microprocessor. It encompasses the transmission overhead costs, excluding idle listening and the startup and shutdown energies. Similarly, in $E_{c r}$ we include the reception overheads. The transmission energy $E_{t}$ consists of the radiated energy and the energy dissipated as heat by the power amplifier.

\subsection{Two Fundamental Requirements for Multihop}

For our initial purpose of finding a fundamental limit of multihop energy-efficiency it suffices to compare single-hop with two-hop, both shown in Figure 1. The relay node $S_{0}$ (which is also a sensing node) serves $K$ other nodes in the two-hop scenario. Note that these $K$ nodes can be positioned in an arbitrary manner as long as communication is possible.

Let us begin with the energy metric comparing the most heavily loaded nodes, denoted $S_{\max }$. In the single-hop case it is the one which must use the largest transmission energy - it can by definition

\footnotetext{
${ }^{1}$ Any network deployment, based on a single node type, in which some nodes are substantially more important than others will suffer from unnecessary sensitivity to node failures, and/or it will be wasting resources.
} 


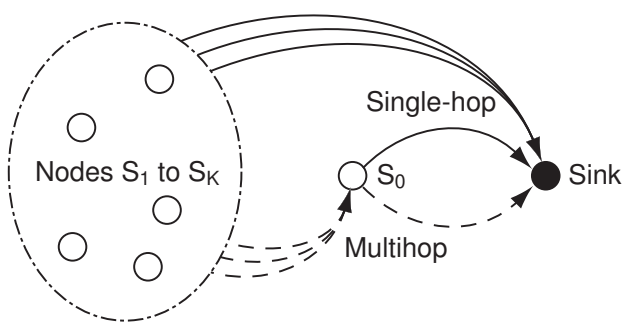

Fig. 1. Simple network scenario where the $K$ nodes $S_{1} \ldots S_{K}$ can transmit directly to the sink (solid lines) or use node $S_{0}$ as a relay and form a two-hop structure (dashed lines). The placement of nodes $S_{1} \ldots S_{K}$ is otherwise unconstrained. Node $S_{0}$ always transmits directly to the sink.

not be node $S_{0}$ - and we denote this energy $E_{t_{\max }}$. The total energy consumption of this node is

$$
E_{\mathrm{SH} \max }=E_{c t}+E_{t_{\max }}
$$

where $E_{\mathrm{SHmax}}$ is our energy metric for the single-hop case. In the multihop case $S_{0}$ is the most heavily loaded node and its energy consumption is

$$
E_{\mathrm{MHmax}}=(K+1) E_{c t}+K E_{c r}+K E_{t_{0}}^{\prime}+E_{t_{0}},
$$

where $E_{\mathrm{MHmax}}$ is the energy metric and $E_{t_{0}}^{\prime}$ and $E_{t_{0}}$ represent transmission energies for relayed bits and $S_{0}$ 's bits respectively. Note that for a given end-to-end performance requirement on the bit error rate, the use of multihop increases the requirement on individual hops as compared to the single-hop case ${ }^{2}$. For this reason, $E_{t_{0}}^{\prime}>$ $E_{t_{0}}$ in (2). If multihop is to be preferable by the present metric we must have $E_{\text {SHmax }}>E_{\text {MHmax }}$. By the use of (1) and (2) this is equivalent to

$$
E_{t_{\max }}>K\left(E_{c t}+E_{c r}\right)+K E_{t_{0}}^{\prime}+E_{t_{0}} .
$$

Node $S_{0}$ 's transmit energies per bit, $E_{t_{0}}^{\prime}$ and $E_{t_{0}}$, will depend greatly on the network conditions, but if we assume that they are of negligible size - an assumption that favours multihop - we get

$$
E_{t_{\max }}>K\left(E_{c t}+E_{c r}\right)
$$

as a fundamental requirement for multihop preference. Put in words, the maximum possible saving in transmission energy must be larger than the incurred circuit overhead energy.

Moving to the total network energy metric, we get in the singlehop case

$$
E_{\text {SHtot }}=(K+1) E_{c t}+\sum_{k=0}^{K} E_{t_{k}} .
$$

The same calculation for multihop yields

$$
E_{\mathrm{MHtot}}=(2 K+1) E_{c t}+K E_{c r}+\sum_{k=1}^{K} E_{t_{k}}^{\prime}+K E_{t_{0}}^{\prime}+E_{t_{0}}
$$

where $E_{t_{k}}^{\prime}$ are the transmission energies from the source nodes to the relay $S_{0}$. Again, by neglecting transmission energies in the multihop case we can derive a conservative requirement for multihop

\footnotetext{
${ }^{2}$ In order to get an end-to-end bit error rate of $B$ over two hops the requirement on each hop is $\approx B / 2$ (to be more precise, $B / 1.999$ ).
}

that states that the mean single-hop transmission energy for nodes $S_{1}$ to $S_{K}$ must exceed the per-node circuit energy;

$$
\frac{1}{K} \sum_{k=1}^{K} E_{t_{k}}>E_{c t}+E_{c r} .
$$

This requirement complements the "max requirement" in (4) and if any of (4) and (7) is not fulfilled multihop should be avoided.

The authors of [3] list the circuit energy and maximum transmit energy for five relevant sensor node radios $^{3}$. We note that, by using (4), if $K=1$, only the $\mu$ AMPS- 1 radio has any chance of saving energy by the use of multihop. If $K=2$ none of the radios will benefit energy-wise from multihop even at maximum transmission distances. Considering this result, it is noteworthy that our approximations favour multihop in several respects:

- the transmission energy $E_{t_{0}}$ was neglected

- the routing energy consumption of multihop was not included

- the ignored startup/shutdown energies, and the ignored cost for idle listening, are all larger in the multihop case

- the maximum transmit energies from [3] are according to the authors generously high

- we restricted ourselves to a single relay, that is two-hop

- central nodes in sensor networks can often be expected to serve more than two nodes even in the simple two-hop case

\section{IN WHICH TRANSMISSION ENVIRONMENTS IS MULTIHOP THE PREFERRED CHOICE?}

Judging from the energy consumption given in [3] it seems that the use of multihop presently can not be motivated by energy-efficiency but only by limited radio range. However, the development of energyefficient and power-aware hardware is constantly progressing, as is the research on efficient transmission techniques, and we expect the barrier in (4) to be broken sooner or later. Assuming this, we now turn to the environmental and system requirements for multihop. In other words, what are the conditions in terms of path losses, fading characteristics, performance requirements, etc., that may render multihop the most energy-efficient alternative?

The required per-hop transmission energy is affected by several factors which we summarise in the expression

$$
E_{t}=\frac{L M N_{f} N_{0}}{\gamma G} f_{\mathcal{T}, \mathcal{F}}(B),
$$

where $L$ is the path loss, $M$ is the link safety margin, $N_{f}$ is the receiver noise figure, $N_{0}$ is the ambient noise power spectral density, $\gamma$ is the power amplifier efficiency, $G$ is the combined gain of the transmit and receive antennas, $f_{\mathcal{F}, \mathcal{T}}(B)$ is the required signal-tonoise ratio per bit corresponding to transmission technique $\mathcal{T}$, fading characteristics $\mathcal{F}$ and target bit error rate $B$.

\subsection{Threshold Path Loss}

We define the boundaries at which multihop may become more energyefficient than single-hop by replacing the inequalities in (4) and (7) with equalities. By the use of (8) we can find the threshold path losses corresponding to the boundaries,

$$
\begin{aligned}
\tilde{L}_{\text {max }} & =K\left(E_{c t}+E_{c r}\right) \frac{\gamma G}{M N_{f} N_{0} f_{\mathcal{T}, \mathcal{F}}(B)} \\
\tilde{L}_{\text {mean }} & =\left(E_{c t}+E_{c r}\right) \frac{\gamma G}{M N_{f} N_{0} f_{\mathcal{T}, \mathcal{F}}(B)}
\end{aligned}
$$

${ }^{3}$ These are the $2.4 \mathrm{Kbps}$ RFM TR1000, the $115.2 \mathrm{Kbps}$ RFM TR1000, $\mu$ AMPS-1, Cisco Aironet 350, and Atheros ISSCC02. 
The threshold path loss is attractive because of its general character. For instance, consider a certain type of wireless sensor network that is expected to operate under conditions with path losses that are greater than $\tilde{L}$. Then the use of multihop will be more energy efficient than the use of single-hop regardless of whether the path loss is caused mainly by walls in an indoor environment or is purely due to distance in a free-space situation.

\subsection{Important Assumptions and Parameter Values}

In order not to bias the comparison unjustly it is essential to consider a wide range of realistic system parameters and environmental assumptions (within the model framework used). The results could be misleading if restrictive assumptions or only one set of specific parameters where used. Unfortunately, we can not cover all possible scenarios. Luckily, the impact of all factors, except $f_{\mathcal{T}, \mathcal{F}}(B)$, in (9) is easily quantified. For instance, an improvement in circuit energy efficiency by a factor of ten lowers the threshold by a factor of ten, that is $10 \mathrm{~dB}$. This would enlarge the region in which a twohop strategy outperforms a single-hop strategy. As a baseline in the comparison we use $K=3, E_{c t}=E_{c r}=1 \mu \mathrm{J}, \gamma=0.35, G=1$, $M=10, N_{f}=5$ and $N_{0}=-204 \mathrm{dBJ}$ (corresponding to $290 \mathrm{~K}$ thermal noise).

When it comes to $f_{\mathcal{T}, \mathcal{F}}(B)$ we will investigate two different target bit error rates, $B=10^{-3}$ and $B=10^{-5}$, for uncoded coherent binary phase shift keying (BPSK). Another important issue concerns the fading channel characteristics, more precisely the degree of fading which can vary greatly between different environments. We therefore include the degree of multipath fading via the Nakagami$\mathrm{m}$ fading model, assuming symbol-independent fading and perfect channel information at the receiver. The Nakagami-m fading model includes a fading figure $m$ ranging from $1 / 2$ to infinity. It is very well motivated both theoretically [6] and empirically [7]. Being established as one of the standard short-term fading models together with the Rayleigh and Rice models, it has been used several times in order to efficiently incorporate the degree of fading into calculations, see for example [8]. Moreover, it contains the Rayleigh model as a special case, $m=1$, and can also accurately approximate the Rice model [6] as well as a static channel, $m \rightarrow \infty$. Different values of $m$ can thus be related to different sensor network and deployment scenarios where certain fading conditions are expected.

\subsection{Very Large Path Losses Required for Multihop}

In Figure 2 the threshold path losses in (9) are displayed for different fading figures $m$ and different target bit error rates $B$. It is evident that the maximum and mean path losses almost always must exceed $100 \mathrm{~dB}$, and quite often even $110 \mathrm{~dB} .{ }^{4}$ The exceptional case is the most unrealistic - the requirement $B=10^{-5}$ in pure Rayleigh fading - and requires "only" an $85 \mathrm{~dB}$ path loss. It should be kept in mind that the thresholds in Figure 2 are conservative as all the transmission costs for multihop are neglected, as is the energy cost associated with multihop routing.

\section{TWO-DIMENSIONAL MULTIHOP NETWORKS}

The simple network structure in Figure 1 served the purpose of establishing some basic limits regarding the energy-efficiency of multihop, but it does not properly capture the scaling effects in twodimensional networks. In such networks, the nodes closest to the

\footnotetext{
${ }^{4}$ We remind the reader that these threshold path losses can be shifted significantly up or down, $\pm 15 \mathrm{~dB}$ say, depending on the parameters in (9).
}

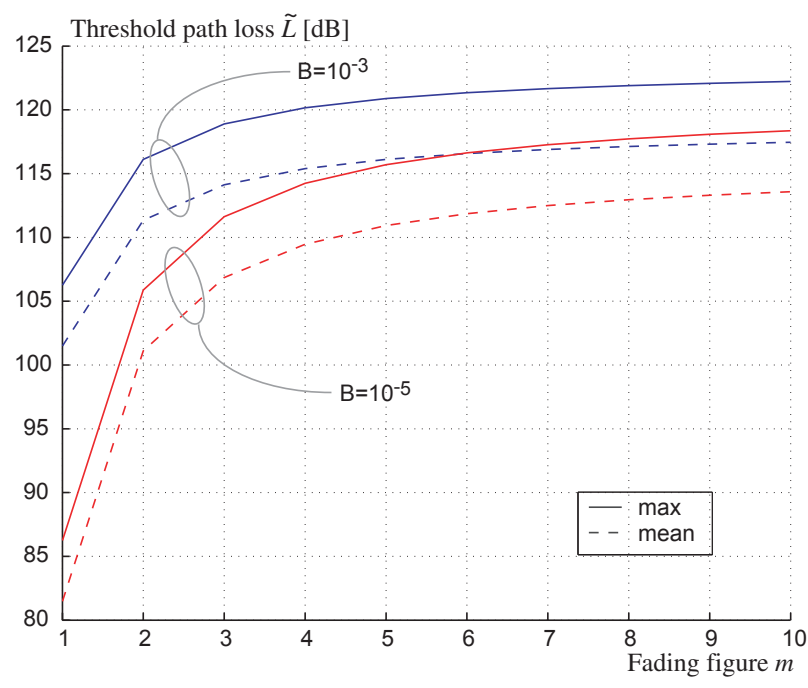

Fig. 2. The threshold path losses $\tilde{L}_{\max }$ (solid) and $\tilde{L}_{\text {mean }}$ (dashed) for different fading environments - different degree of fading - in the two-hop scenario depicted in Figure 1. Results for two target bit error rates, $B=10^{-3}$ and $B=10^{-5}$ are shown.

sink can become very strained as they must serve a large number of nodes by relaying their data. For simplicity, let us consider a square and uniform $2 K \times 2 K$ network with the sink placed centrally.

\subsection{Total Network Energy}

If all $(2 K)^{2}$ nodes transmit directly to the sink, they will consume a total energy of

$$
E_{\text {SHtot }}=(2 K)^{2} E_{c t}+\sum_{k=1}^{(2 K)^{2}} E_{t k},
$$

while a multihop strategy in which all transmits to their closest neighbour (that is closer to the sink) will lead to ${ }^{5}$

$E_{\mathrm{MHtot}}=\frac{2 K}{3}\left[\left(4 K^{2}+3 K-1\right) E_{c t}+\left(4 K^{2}-3 K-1\right) E_{c r}\right]+E_{t_{\mathrm{all}}}$,

where $E_{t_{\text {all }}}$ is the sum of all $\left(\left(8 K^{3}+6 K^{2}-2 K\right) / 3\right.$ transmit energies. We continue our conservative approach and neglect $E_{t_{\text {all }}}$ in the comparison. By the use of (8), (10) and (11) we find that

$$
\tilde{L}_{\text {mean }}=\left(\frac{2}{3} K-\frac{1}{2}-\frac{1}{6 K}\right)\left(E_{c t}+E_{c r}\right) \frac{\gamma G}{M N_{f} N_{0} f_{\mathcal{T}, \mathcal{F}}(B)} .
$$

\subsection{Energy in the Most Heavily Loaded Node}

Consider now the most heavily loaded nodes. In the single-hop case we simply have, as in (1),

$$
E_{\mathrm{SHmax}}=E_{c t}+E_{t_{\max }} .
$$

${ }^{5}$ Divide the network of $(2 K)^{2}$ nodes into 4 quadrants and consider an arbitrary quadrant. The total circuit energy can be computed by considering hop by hop what each node's sensor data requires for its entire route. The transmission from the inmost node requires $E_{c t}$. In the nodes one hop away from the inmost node, the requirement is $2 E_{c t}+E_{c r}$ for each node (3 nodes on this level). Two hops away, the requirement is $3 E_{c t}+2 E_{c r}$ for each node ( 5 nodes on this level), and so on. Summing all terms until the $K$ th layer in each quadrant and multiplying by 4 , we find the total energy as in (11). 


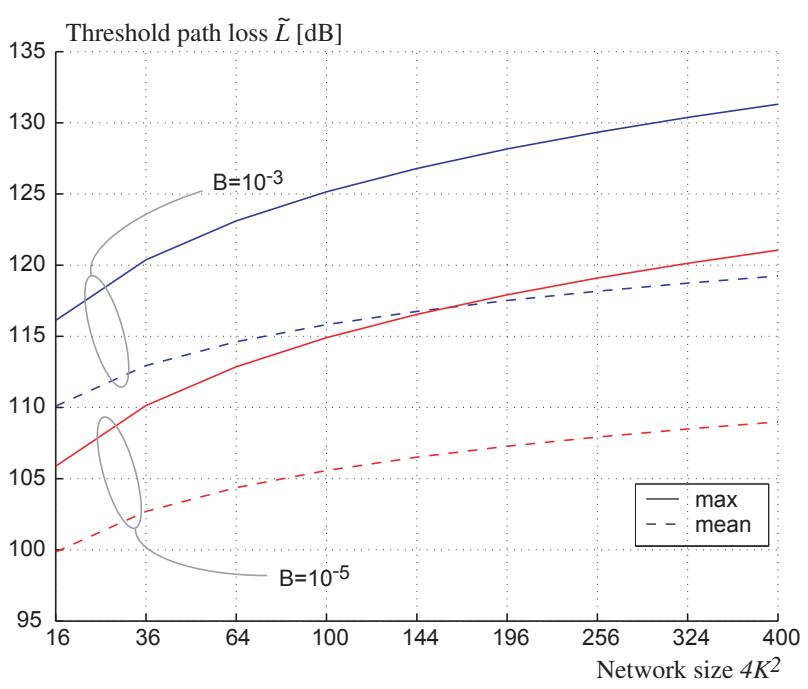

Fig. 3. The threshold path losses $\tilde{L}_{\text {max }}$ (solid) and $\tilde{L}_{\text {mean }}$ (dashed) for a two-dimensional network consisting of $4 K^{2}$ nodes. Results for two target bit error rates, $B=10^{-3}$ and $B=10^{-5}$ are shown.

In the multihop case, the four innermost nodes must each forward the data from $K^{2}-1$ nodes and therefore consume the energy

$$
E_{\mathrm{MH} \max }=K^{2} E_{c t}+\left(K^{2}-1\right) E_{c r}+\sum_{k=1}^{K^{2}-1} E_{t k}^{\prime}+E_{t_{0}}
$$

Our conservative threshold path loss in this case becomes

$$
\tilde{L}_{\max }=\left(K^{2}-1\right)\left(E_{c t}+E_{c r}\right) \frac{\gamma G}{M N_{f} N_{0} f_{\mathcal{T}, \mathcal{F}}(B)} .
$$

Shown in Figure 3 are the threshold path losses (12) and (15) for different network sizes assuming Nakagami $m=2$ fading and using the same parameters as before. It can be immediately observed that the required mean or maximum losses are prohibitively large. A naive multihop strategy for a large network wastes a lot of energy as compared to a simple single-hop strategy. It would take a dramatic improvement in circuit energy consumption for a qualitative change of conclusions (and remember that our basic case here still assumes quite efficient circuitry using $1 \mu \mathrm{J}$ per bit). Concerning advanced multihop schemes we note that the authors of [9] find that not even an optimised scheme can alleviate the asymmetric load problem.

\section{WHAT ABOUT DATA FUSION AND INTERFERENCE?}

One could argue that node $S_{0}$, in some applications, will be able to perform some sort of data fusion or data aggregation and thereby save energy by reducing the number of bits to send. However, even if such a scheme would be perfect, in the sense that $K+1$ bits could be reduced to a single bit, this would only have marginal impact on the threshold $\tilde{L}_{\text {max }}$ in (9). For example, if $K=3$ as in Figure 2 the threshold would be lowered by less than $2 \mathrm{~dB}$. And then we have ignored the processing energy.

Transmit powers in a single-hop network will exceed those in a multihop network and this could seemingly lead to higher interfer- ence levels. We are however considering many-to-one networks in which some kind of synchronisation helps avoid interference from other nodes. A single-hop network will be much easier to synchronise than its multihop counterpart.

\section{CONCLUSIONS}

It is clear that even two hops in a wireless sensor network is simply one too many when our aim is maximum network life-time, be it defined in terms of the time when the first node runs out of power or total network energy. We hereby confirm the suspicions raised by Min and Chandrakasan [3] in greater generality. It should be emphasised that although the topology of Figure 1 may seem to be a special case, it is in fact quite general when our concern is the most heavily loaded node. For different multihop routing protocols, there will always be at least one node that relays data from at least one other node. By choosing $K$ as the number of nodes which are relayed through the most heavily loaded node we can investigate any routing protocol by the topology in Figure 1.

Finally, it can be observed that our analysis here speaks in favour of deploying hierarchical structures (a research topic receiving increased attention) where low level nodes communicate with higher layers via single-hop only (a strategy seldom applied). A challenging problem lies in devising a self-organising structure for building such hierarchies effectively and energy-efficiently.

\section{REFERENCES}

[1] A. Woo, T. Tong and D. Culler "Taming the underlying challenges of reliable multihop routing in sensor networks", Proc. of the 1st Int. Conf. on Embedded Networked Sensor Systems, pp. 14-27, Nov. 2003

[2] R. Min, M. Bhardwaj, S. Cho, N. Ickes, E. Shih, A. Sinha, A. Wang and A. Chandrakasan, "Energy-centric enabling technologies for wireless sensor networks", IEEE Wireless Comm., pp. 28-39, Aug. 2002

[3] R. Min and A. P. Chandrakasan, "Top five myths about the energy consumption of wireless communication", ACM Mobile Comp. and Comm. Rev., vol. 6, no. 4, pp. 65-67, 2002

[4] R. Min and A. Chandrakasan, "A framework for energy-scalable communication in high-density wireless networks", Proc. 2002 Int. Symp. on Low Power Elec. and Design, pp. 36-41, 2002

[5] L. C. Zhong, J. M. Rabaey, A. Wolisz, "Does proper coding make single hop wireless sensor networks reality: the power consumption perspective", Proc. IEEE Wireless Comm. and Networking Conf., vol. 2, pp. 664-669, Mar. 2005

[6] S. A. Abbas and A. U. Sheikh, "A geometric theory of Nakagami fading multipath mobile radio channel with physical interpretations," Vehicular Tech. Conf., vol. 2, p. 637-641, 1996

[7] H. Suzuki, "A statistical model for urban radio propogation", IEEE Trans. on Comm., vol. 25, no. 7, p. 673-680, Jul. 1977

[8] U. Charash, "Reception through Nakagami fading multipath channels with random delays", IEEE Trans. on Comm., vol. 27, no. 4, pp. 657-670, Apr. 1979

[9] M. Perillo, C. Zhao and W. Heinzelman, "On the problem of unbalanced load distribution in wireless sensor networks", GlobeCom Workshops, pp. 74-79, Nov. 2004 